\title{
Taxonomic distinctness and diversity measures: responses in marine fish communities
}

\author{
Stephen J. Hall ${ }^{1, *}$, Simon P. Greenstreet ${ }^{2}$ \\ ${ }^{1}$ School of Biological Sciences, Flinders University of South Australia, GPO Box 2100, Adelaide, South Australia 5001, Australia \\ ${ }^{2}$ Marine Laboratory, PO Box 101, Victoria Rd, Aberdeen AB11 9DB, Scotland, UK
}

\begin{abstract}
In a recent paper Warwick \& Clarke (1995: Mar Ecol Prog Ser 129:301-305) introduced 2 new measures of community diversity, $\Delta$ and $\Delta^{*}$ These measures differ from more conventional diversity indices by incorporating the taxonomic relatedness of species into their calculation. In this paper we compare temporal trends in $\Delta$ and $\Delta^{*}$ with more conventional diversity measures for the demersal fish community of the northern North Sea. We show that, contrary to the findings for benthic invertebrates, indices that incorporate taxonomic distinctness show identical time trends to conventional measures. This, perhaps, suggests that when perturbations are sufficiently large to affect conventional indices of diversity, taxonomic diversity indices may already have reached levels from which they march in step with conventional indices.
\end{abstract}

KEY WORDS: Biodiversity - Taxonomic distinctness - Environmental perturbation - Fish communities

\section{INTRODUCTION}

Assemblages with the same species diversity may comprise species which are all closely related to each other taxonomically or be of species that are only distantly related. Conventional diversity indices such as the Shannon index $H^{\prime}$ (Pielou 1975) take no account of this possibility. Noting this fact, Warwick \& Clarke (1995) recently proposed 2 new measures of community diversity, $\Delta$ and $\Delta^{*}$, which incorporate taxonomic relatedness into their calculation. The authors describe $\Delta$ as an index of taxonomic diversity because it is empirically related to the Shannon's species diversity $H^{\prime}$, but has an added component of taxonomic separation. In contrast, $\Delta^{*}$ can be thought of purely as a measure of taxonomic distinctness, without the contribution from species diversity.

The theoretical basis for the addition of a taxonomic element to community analyses rests on the observation that, in grossly perturbed environments, benthic communities are kept at an early successional stage with relatively few species, many of which are closely related. In contrast, less perturbed benthic communities tend to comprise a range of more distinct species

•E-mail: stephen.hall@flinders.edu.au belonging to many different phyla. Warwick \& Clarke (1995) also observe that, for benthic communities subject to moderate levels of anthropogenic disturbance, univariate measures of species diversity often do not change, yet changes in community composition can be revealed by multivariate analysis of species abundance data (e.g. Gray et al. 1990, Warwick \& Clarke 1991, Dawson Shepherd et al. 1992). This latter observation implies that, at moderate levels of disturbance, while particular species abundances change, the overall distribution of individuals among species is preserved. These 2 sets of observations led Warwick \& Clarke (1995) to propose that this apparent homeostasis in diversity is achieved through shifts in the hierarchy of relatedness above the species level, and that these shifts might be revealed by $\Delta$ and $\Delta^{\circ}$.

Warwick \& Clarke (1995) demonstrated that $\Delta$ and $\Delta^{*}$ behave in intuitively reasonable ways when small example communities are constructed. Furthermore, in a real situation around an oilfield where the Shannon index $H^{\prime}$ remained constant, a continuous decrease in the taxonomic distinctness of a marine invertebrate assemblage could be detected along a gradient of increasing environmental contamination. In the same paper the authors also examined the effects of sample size on the behaviour of the indices 
and showed that, unlike most other diversity measures, there was no systematic bias at low sample sizes - a highly desirable property for any index.

Based on these preliminary analyses it would appear that including taxonomic relatedness into diversity measures may provide more robust and sensitive indices of community perturbation. It can also be legitimately argued that such measures more truly reflect our notions of 'biodiversity', since a community of many closely related species would be viewed by most people as being less diverse than one in which the same number of species is more distantly related.

Previously, we have described changes in the structure of the demersal fish community of the northern North Sea between the years 1929-1953 and 1980-1993 (Greenstreet \& Hall 1996). Our goal was to examine the possible effects that fisheries activity may have had. We found that species diversity (as revealed by indices such as Shannon's exp $H^{\prime}$ ) varied among years, but that trends over time were generally inconsistent with a fisheries effect. In contrast, multivariate analysis revealed differences among areas and times, suggesting that communities had changed, perhaps as a result of fisheries activity. Here we extend these analyses using $\Delta$ and $\Delta^{*}$ to examine whether there have been shifts in the taxonomic relatedness of the assemblage comparable to those observed by Warwick \& Clarke (1995).

\section{METHODS AND RESULTS}

Our fish data are from demersal trawl surveys conducted by Scottish research vessels during 2 time periods: 1929-1953 (hereafter referred to as the Archive data set) and 1980-1993, the August Ground Fish Survey (AGFS). Unfortunately, data between these 2 periods cannot be included owing to changes in data collection procedures. To compare the 2 time periods, data from the Archive data set were restricted to samples taken between July and September. Full details of collection procedures and the areas covered by the surveys are given in Greenstreet \& Hall (1996). The combined data set comprises 76 species divided among 61 genera, 13 families and 13 orders. For this analy-
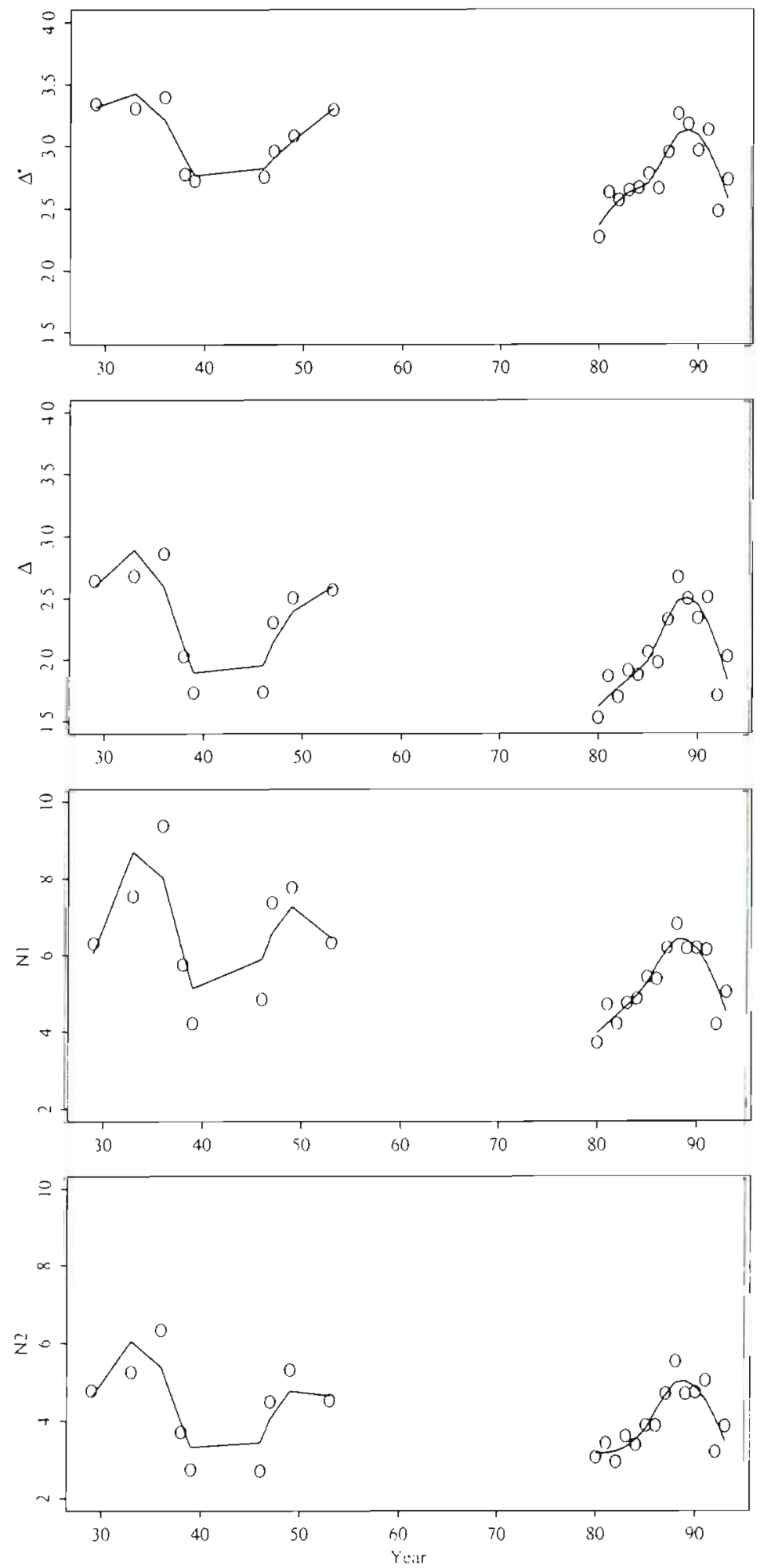

Fig. 1 Time series plots for 2 standard diversity indices, Hill's N1, the exponential of the Shannon index (exp $H^{\prime}$ ), and Hill's N2, the reciprocal of Simpson's index $(1 / D)$, and 2 indices incorporating taxonomic relatedness, $\Delta$ and $\Delta^{*}$ Lines indicate non-parametric smoothed trends fitted to each survey series separately. (Sne text for further explanation) 
sis, we have combined all data for a given year to obtain a single estimate for each of 4 indices, $\Delta$ and $\Delta^{*}$ as described above and Hill's N1 and N2, the exponential of the Shannon index (exp $\left.H^{\prime}\right)$ and the reciprocal of Simpson's index $(1 / D)$, respectively.

The procedure for calculating $\Delta$ and $\Delta^{*}$ is repeated here for completeness. Let $x$, denote the abundance of the $i$ th species $(i=1, \ldots, S)$ and $w_{i j}$ denote the weighting given for the taxonomic distinctness between species $i$ and species $j$. We distinguished fish at 4 taxonomic levels, species, genus, family and order, and assigned taxonomic weights in the following manner: for species in the same genus, $w=1$; for species in different genera, but the same family, $w=2$; for species in different families, but the same order, $w=3$; for species in different orders $w=4$.

Armed with taxonomic weights and species abundances, $\Delta$ and $\Delta^{*}$ are defined as:

$$
\Delta=\frac{\sum \sum_{i<j} w_{i j} x_{i} x_{j}+\sum_{i} 0 \cdot x_{i}\left(x_{i}-1\right) / 2}{\sum \sum_{i<j} x_{i} x_{j}+\overline{\sum_{i}} x_{i}\left(x_{i}-1\right) / 2}
$$

and

$$
\Delta^{*}=\frac{\sum \sum_{i<j} w_{i j} x_{i} x_{j}}{\sum \sum_{i<j} x_{j} x_{j}}
$$

As in the original published description of the index, the null term in the numerator of Eq. (1) is included to emphasise that the weight for individuals of the same species is taken to be zero.

Fig. 1 shows the time series for the 4 indices, with a non-parametric smoothed trend fitted to each survey series separately. Smoothing parameters were identical for all graphs. Although there are trends apparent in each time series, the key point with respect to this analysis is that all indices behave in a broadly similar manner within each data series. The only marked departure between $\Delta$ and $\Delta^{*}$ and $N 1$ and N2 is that the latter 2 indices suggest lower values for diversity at the 2 ends of the Archive time series. Taken overall, however, the indices track each other to a much greater degree than that observed by Warwick \& Clarke (1995) for a benthic community.

\section{DISCUSSION}

The close correlation between the 2 classes of index for our demersal fish assemblage is in marked contrast to that obtained by Warwick \& Clarke (1995) for the benthos. Although we see changes over the 60 yr our data span, both taxonomic distinctness and the distribution of individuals among species march in step. How can we account for this difference in behaviour? One contrast between our analysis and that of Warwick \& Clarke (1995) is that in our case conventional diversity indices did differ between samples (years) rather than remain constant. It is possible, therefore, that the taxonomic shifts revealed by the delta indices occur at lower levels of perturbation, where conventional diversity is insensitive to change, and that it is only at higher levels that the 2 kinds of measure behave similarly. In other words, when perturbations are sufficiently large to have shifted conventional diversity indices, indices incorporating taxonomic relatedness will already have changed and will thereafter track conventional measures. If this hypothesis is true, it implies that taxonomic relatedness indices may indeed be more sensitive at detecting the early stages of community change. At present such an hypothesis is little more than speculation, but it is certainly amenable to experimental test. Another explanation, however, may be related to the fact that, unlike benthic analyses which involve many species from a number of major taxa, we only have data for fish. This more restricted taxonomic range may constrain the behaviour of the delta indices and give them less scope to depart from the trends shown by conventional measures. Further analysis of the behaviour of these statistics will also be required to examine this possibility.

Because all indices correlate closely for the North Sea fish assemblage, in one sense we seem to gain no additional insight by using one index over another. It must be borne in mind, however, that these new indices measure another component of community structure that is of intrinsic interest. Their use with these data suggests that, as with conventional diversity, while there are trends over time within the 2 survey data sets, there is little indication of marked changes in the taxonomic hierarchy ('biodiversity') that might be associated with, for example, increases in fishing effort.

\section{LITERATURE CITED}

Dawson Shepherd AR, Warwick RM, Clarke KR, Brown BE (1992) An analysis of fish community responses to coral mining in the Maldives. Environ Biol Fish 33:367-380

Gray JS, Clarke KR, Warwick RM, Hobbs G (1990) Detection of initial effects of pollution on marine benthos: an examination from the Ekofisk and Eldfisk oilfields, North Sea. Mar Ecol Prog Ser 66:285-299

Greenstreet SPR, Hall SJ (1996) Fishing and groundfish assemblage structure in the northwestern North Sea: an analysis of long-term and spatial trends. J Anim Ecol 65: $577-598$

Pielou EC (1975) Ecological diversity. J Wiley \& Sons, New York

Warwick RM, Clarke KR (1991) A comparison of some methods for analysing changes in benthic community structure. J Mar Biol Assoc UK 7 1:225-244

Warwick RM, Clarke KR (1995) New 'biodiversity' measures reveal a decrease in taxonomic distinctness with increasing stress. Mar Ecol Prog Ser 129:301-305

Submitted: August 25, 1997; Accepted: March 30, 1998

Proofs received from author(s): April 27, 1998 